\title{
Statyba
}

\section{MIXED AND COMPATIBLE FINITE ELEMENTS IN THE ANALYSIS PROBLEM OF ELASTOPLASTIC STRUCTURES}

\section{S. Kalanta}

To cite this article: S. Kalanta (1996) MIXED AND COMPATIBLE FINITE ELEMENTS IN THE ANALYSIS PROBLEM OF ELASTOPLASTIC STRUCTURES, Statyba, 2:5, 29-43, DOI: 10.1080/13921525.1996.10531546

To link to this article: https://doi.org/10.1080/13921525.1996.10531546

曲 Published online: 26 Jul 2012.

Submit your article to this journal $₫$

Џ Article views: 66 


\section{СМЕШАННЫЕ И СОВМЕСТНЫЕ КОНЕЧНЫЕ ЭЛЕМЕНТЫ В ЗАДАЧАХ АНАЛИЗА УПРУГОПЛАСТИЧЕСКИХ КОНСТРУКЦИЙ}

\section{С. Каланта}

\section{1. Введене}

Обпие математические модели задач анализа напряхенно-деформированного состояния (НДС) и огтимизации упругопластического тела разработаны в [1,2]. Настояпая статья посвящена совершенствованию и развитию двойственных конечноэлементных математических моделей задач анализа НДС в состояния до пластического разрушения. Соблюдение двойственньх связей статических и геометрических уравнений позволяет упростить математические модели и алгоритмы решения задач [3]. Это особенно важно для задач оптимизации при ограниченных перемещениях. Обнчно в двойственных формулировках задач [4-6 и др.] проблема учета возможных разрнвов перемещений не рассматривается, т.е. негласно принимается предположение о неразрывности полей перемещений, которое не всегда соответствует действителњному состоянию упругопластических хонструхций. Разрьвы схоростей перемещений обычно учитываются только в кинематической постановке задач предельной нагрузки [7-9 и др.], построеннбх на основе кинематической теоремы предельного равновесия независимо от статичесхой постановхи (двойственность не соблюдается). Условия неразрнвности перемещений принимаются даже в некоторнх кинематических хонечноэлементных постановках задач. Однахо такие постановхи непритодня для анализа хонструкпий, в которнх образуются поверхности сосредоточенных пластических деформаций.

В работах $[3,10]$ построены обшие двойственнте математические модели задач анализа НДС и оптимизации упругопластических конструкпий с учетом возможных разрнвов перемещений. На основе этих исследований в [3] построены двойственные равновесные конечноэлементные постановхи задач анализа. Они позволяпо определить верхние оценхи напряжений и перемещений. Однако на основе смешанного энергетического функпионала с применением смешанншх конечншх элементов и метода преобразований Јагранжа можно построить и вторую пару двойственных конечноэлементных математических моделей задачи. Вместе с равновесными моделями они позволили бы осуществить двухсторонший анализ и оценить точность численных результатов расчета упругопластических конструкций. Построение совместных и смешанғых конечноэлементншх математических моделей задачи анализа НДС, учитьвающих возможные разрывы перемещений, и является основной целью настоящих исследований.

\section{2. Общие математические модели}

Рассматривается тело (обобщенная модель конструкции), геометрические и физические параметры, условия опирания и функция $C(\mathbf{x})$ параметра текучести которого заданы. Тело подвергается действио внешней нагрузки $\mathbf{F}(\mathbf{x})$, распределенной на поверхности $S_{f}$, а также начальных деформаций $\varepsilon_{0}(\mathbf{x})$ и перемещений $\mathbf{u}_{0}(\mathbf{x})$, заданных в объеме $V$ и на поверхности $S_{u}$ 
соответственно. Дискретная модель тела строится расчленением его на $s$ конечных элементов объема $V_{k}$. Напряженно-деформированное состояние $k$-го элемента описьвается векторфункциями напряжений $\sigma_{k}(\mathbf{x})$, перемещений $\mathbf{u}_{k}(\mathbf{x})$ и деформаций $\varepsilon_{k}(\mathbf{x})$. Функпии, относящцеся $\mathbf{x}$ смежным конечным элементам, разделенным поверхностью $S_{t}$, обозначаются знаками " + " и “ - ". Пусть номера конечных элементов $k=1,2, \ldots, s$ образуют множество $\mathscr{K}$, а совокупность номеров

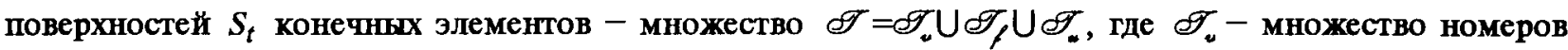
внутренних межэлементнбх поверхностей; $\mathscr{E}_{f}, \mathscr{E}_{-}-$множества номеров граничных, принадлежаших $S_{f}$ и $S_{u}$, поверхностей элементов. Пусть $\mathscr{E}_{f}=\mathscr{E} \mathscr{E}_{f}$.

Рассмотрим задачи определения полнбх и остаточнњх параметров НДС тела. Для построения математической модели смешанной постановки задач воспользуемся теоремой о минимуме дополнительной энергии. В терминах полньх напряхений она внрахается следующей задачей квадратичното программирования [3]:

$$
\sum_{k} \frac{1}{2} \int_{V_{k}} \sigma_{k}^{T}(\mathbf{x})\left[d_{k}\right] \sigma_{k}(\mathbf{x}) d V_{k}+\sum_{k} \int_{V_{k}} \sigma_{k}^{T}(\mathbf{x}) \varepsilon_{0 k}(\mathbf{x}) d V_{k}-\sum_{t \in \mathscr{G} . S_{S^{t}}} \int_{0 t}^{T}(\mathbf{x}) \mathbf{p}_{t}(\mathbf{x}) d S_{t} \rightarrow \min
$$

при условиях

$$
\begin{gathered}
\mathbf{p}_{0}\left(C_{k}(\mathbf{x})\right)-\mathbf{f}\left(\sigma_{k}(\mathbf{x})\right) \geq \mathbf{0} \quad \in V_{k}, \quad \mathbf{t}_{0 t}\left(C_{t}(\mathbf{x})\right)-\mathbf{t}_{t}\left(\mathbf{p}_{t}(\mathbf{x})\right) \geq \mathbf{0} \in S_{t}, \quad t \in \mathscr{F} ; \\
-[\mathscr{A}] \sigma_{k}(\mathbf{x})=\mathbf{0} \in V_{k}, k \in \mathscr{O} ; \quad\left[A_{t}\right] \sigma_{t}(\mathbf{x})-\mathbf{p}_{t}(\mathbf{x})=\mathbf{0} \in S_{f}, S_{u}, \quad t \in \mathscr{G}_{f}, \mathscr{O}_{*} ; \quad \mathbf{p}_{t}(\mathbf{x})=\mathbf{F}_{t}(\mathbf{x}) \in S_{f}, \\
{\left[A_{t}\right] \sigma_{t}^{+}(\mathbf{x})-\mathbf{p}_{t}(\mathbf{x})=\mathbf{0}, \quad-\left[A_{t}\right] \sigma_{t}^{-}(\mathbf{x})+\mathbf{p}_{t}(\mathbf{x})=\mathbf{0} \quad \in S_{t}, \quad t \in \mathscr{F}_{\bullet} .}
\end{gathered}
$$

Здесь $[\mathscr{A}],\left[A_{t}\right]$ - отераторь дифференциальньх условий равновесия, статических граничнкх условий и условий сопряжения конечных элементов; $\left[d_{k}\right]$ - матрица податливости элементарнопо объема $\boldsymbol{k}$-то элемента. Неравенства представляют собой условия текучести хонечннх элементов и их поверхностей, а равенства - условия равновесия в объеме элементов и мехду элементами.

Исполъзуя метод мнохителей Лагранжа и формулу Гаусса-Остроградского, задачу анализа полнбх напряхений (1) преобразуем в задачу стапионарности смешанного функщионала:

$$
\begin{aligned}
& =\sum_{k} \frac{1}{2} \int_{V_{k}} \sigma_{k}^{T}(\mathbf{x})\left[d_{k}\right] \sigma_{k}(\mathbf{x}) d V_{k}+\sum_{k} \int_{V_{k}} \sigma_{k}^{T}(\mathbf{x}) \varepsilon_{0 k}(\mathbf{x}) d V_{k}-\sum_{k} \int_{V_{k}} \sigma_{k}^{T}(\mathbf{x})[\mathscr{C}]^{T} \mathbf{u}_{k}(\mathbf{x}) d V_{k}+ \\
& +\sum_{t \in \mathscr{O},} \int_{S_{s}}\left\{\mathbf{u}_{t}^{-}(\mathbf{x})\right\}^{T} \mathbf{F}_{t}(\mathbf{x}) d S+\sum_{t \in \mathscr{G},} \int_{S_{t}} \mathbf{p}_{t}^{T}(\mathbf{x})\left\{\mathbf{u}_{t}^{+}(\mathbf{x})-\mathbf{u}_{t}^{-}(\mathbf{x})\right\} d S_{t}+\sum_{t \in \mathscr{O},} \int_{S_{s t}} \mathbf{p}_{t}^{T}(\mathbf{x})\left\{\mathbf{u}_{t}^{+}(\mathbf{x})-\mathbf{u}_{0 t}(\mathbf{x})\right\} d S_{t}- \\
& -\sum_{k} \int_{V_{k}} \lambda_{k}^{T}(\mathbf{x})\left\{\mathbf{f}_{0}\left(C_{k}(\mathbf{x})\right)-\mathbf{f}\left(\sigma_{k}(\mathbf{x})\right)\right\} d V_{k}-\sum_{t \in \mathscr{G}} \int_{S_{t}} \lambda_{t}^{T}(\mathbf{x})\left\{\mathbf{f}_{0 t}\left(C_{t}(\mathbf{x})\right)-\mathbf{t}_{t}\left(\mathbf{p}_{t}(\mathbf{x})\right)\right\} d S_{t} \rightarrow \text { stac }
\end{aligned}
$$

при условиях

$$
\lambda_{k}(\mathbf{x}) \geq 0 \quad \in V_{k}, \quad k \in \mathscr{K} ; \quad \lambda_{t}(\mathbf{x}) \geq 0 \quad \in S_{t}, \quad t \in \mathscr{F} .
$$

Условиями стационарности этого фунхционала по переменным $\sigma_{k}(\mathbf{x})$ и $\mathbf{p}_{t}(\mathbf{x})$ являются уравнения:

$$
\begin{gathered}
{\left[d_{k}\right] \sigma_{k}(\mathbf{x})+\varepsilon_{0 k}(\mathbf{x})+\left[\nabla \mathbf{f}\left(\sigma_{k}(\mathbf{x})\right)\right]^{T} \lambda_{k}(\mathbf{x})-[\mathscr{A}]^{T} \mathbf{u}_{k}(\mathbf{x})=\mathbf{0} \quad \in V_{k}, \quad k \in \mathscr{K} ;} \\
{\left[\nabla \mathbf{f}_{t}\left(\mathbf{p}_{t}(\mathbf{x})\right)\right]^{T} \lambda_{t}(\mathbf{x})-\mathbf{u}_{t}^{-}(\mathbf{x})+\mathbf{u}_{t}^{+}(\mathbf{x})=\mathbf{0} \in S_{t}, \quad t \in \mathscr{\mathscr { T }} ;} \\
{\left[\nabla \mathbf{f}_{t}\left(\mathbf{p}_{t}(\mathbf{x})\right)\right]^{T} \lambda_{t}(\mathbf{x})-\mathbf{u}_{0 t}(\mathbf{x})+\mathbf{u}_{t}^{+}(\mathbf{x})=\mathbf{0} \in S_{t}, \quad t \in \mathscr{O}_{*} .}
\end{gathered}
$$

Они представляют собой геометрические уравнения конечных элементов, условия совместности

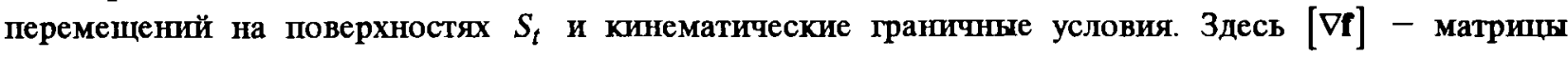


градиентов функаций текучести. Пятый и шестой ингегралы функционала выражают энергию дисситации на поверхностях $S_{t}$.

Для кинематически возможньх функций перемещений, удовлетворяющих условиям (3), функионал , умноженнњй на -1 , выражает полную энергию деформаций упругопластического тела. Поэтому задача стационарности (2) может быть преобразована в следующую задачу:

$$
\begin{aligned}
& -\sum_{k} \frac{1}{2} \int_{V_{k}} \sigma_{k}^{T}(\mathbf{x})\left\{d_{k}\right] \sigma_{k}(\mathbf{x}) d V_{k}-\sum_{k} \int_{V_{k}} \lambda_{k}^{T}(\mathbf{x})\left[\nabla \mathbf{f}\left(\sigma_{k}(\mathbf{x})\right)\right] \sigma_{k}(\mathbf{x}) d V_{k}-\sum_{t \in \mathscr{O}} \int_{S_{t}} \lambda_{t}^{T}(\mathbf{x})\left[\nabla \mathbf{f}_{t}\left(\mathbf{p}_{t}(\mathbf{x})\right)\right] \mathbf{p}_{t}(\mathbf{x}) d S_{t}+ \\
& +\sum_{t \in \mathscr{F},} \int_{S_{f}}\left\{\mathbf{u}_{t}^{-}(\mathbf{x})\right\}^{T} \mathbf{F}_{t}(\mathbf{x}) d S-\sum_{k} \int_{V_{k}} \lambda_{k}^{T}(\mathbf{x})\left\{\mathbf{f}_{0}\left(C_{k}(\mathbf{x})\right)-\mathbf{f}\left(\sigma_{k}(\mathbf{x})\right)\right\} d V_{k}-\sum_{t \in \mathscr{T}} \int_{S_{t}} \lambda_{t}^{T}(\mathbf{x})\left\{\mathbf{f}_{0 t}\left(C_{t}(\mathbf{x})\right)-\mathbf{f}_{t}\left(\mathbf{p}_{t}(\mathbf{x})\right)\right\} d S_{t} \rightarrow \max
\end{aligned}
$$

при условиях (3) и $\lambda_{k}(\mathbf{x}) \geq 0 \in V_{k}, k \in \mathscr{K} ; \lambda_{t}(\mathbf{x}) \geq \mathbf{0} \in S_{t}, t \in \mathscr{T}$. Она отвечает теореме о минимуме полной энергии деформаций [3] и, как и зазача (2), позволяет определить распределение напряжений и перемещений в состояпи до пластического разрушения тела. Неизвестными в ней явлпотся статические и кинематические величины. Поэтому она назнвается общей математической модельо смешанной постановки задачи анализа НДС.

Если в качестве основных неизвестных после предварителыного решения задачи теории упругости принять остатопные перемещения и напряжения, то вариационная постановка задачи анализа упругопластической конструкиии представляется задачей стационарности фунхционала:

$$
\begin{aligned}
& \mathbb{F}_{-}=\sum_{k} \frac{1}{2} \int_{V_{k}} \sigma_{n k}^{T}(\mathbf{x})\left[d_{k}\right] \sigma_{r k}(\mathbf{x}) d V_{k}-\sum_{k} \int_{V_{k}} \sigma_{r k}^{T}(\mathbf{x})[\mathscr{O A}]^{T} \mathbf{u}_{r k}(\mathbf{x}) d V_{k}+ \\
& +\sum_{t \in \mathscr{G},} \int_{S_{t}} \mathbf{p}_{r t}^{T}(\mathbf{x})\left\{\mathbf{u}_{n t}^{+}(\mathbf{x})-\mathbf{u}_{n t}^{-}(\mathbf{x})\right\} d S_{t}+\sum_{t \in \mathscr{G} .} \int_{S_{w}} \mathbf{p}_{n t}^{T}(\mathbf{x}) \mathbf{u}_{n t}^{+}(\mathbf{x}) d S-\sum_{k} \int_{V_{k}} \lambda_{k}^{T}(\mathbf{x})\left\{\mathbf{f}_{0}\left(C_{k}(\mathbf{x})\right)-\mathbf{f}\left(\sigma_{e k}(\mathbf{x})+\sigma_{r k}(\mathbf{x})\right)\right\} d V_{k}- \\
& -\sum_{t \in \mathscr{G}} \int_{S_{t}} \lambda_{t}^{T}(\mathbf{x})\left\{\mathbf{f}_{0 t}\left(C_{t}(\mathbf{x})\right)-\mathbf{f}_{t}\left(\mathbf{p}_{e t}(\mathbf{x})+\mathbf{p}_{n t}(\mathbf{x})\right)\right\} d S_{t} \rightarrow \operatorname{stac}
\end{aligned}
$$

при условиях

$$
\lambda_{k}(\mathbf{x}) \geq 0 \quad \in V_{k}, \quad k \in \mathscr{K} ; \quad \lambda_{t}(\mathbf{x}) \geq 0 \quad \in S_{t}, \quad t \in \mathscr{F},
$$

которая может быть преобразована в задачу минимизации полной энергии остаточнњх деформаций при ограничениях, определяющих область кинематически возможних функий перемещений. Здесь индексом $е$ обозначены параметры упругого расчета конструкции, а индексом $r$ - остаточные параметрн. Третий и четвертый члены функпионала в выражают энергию диссипашии на пластических поверхностях конечных элементов. Представленные вариационные ностановхи далее используются для построения двойственной пары дискретных математическмх моделей задач.

\section{3. Дискретные математнческие модели}

\section{1. Задача анализа полнхх перемещений н напряжений}

Аппроксимирующие функции. Для дискретизация задачи (2) применяются смешанные конечные элементы. Внбираются аппроксимирующие функции

$$
\begin{aligned}
& \sigma_{k}(\mathbf{x})=\sum_{i=1}^{\xi}\left[H_{k i}(\mathbf{x})\right] \sigma_{k i}=\left[H_{k}(\mathbf{x})\right] \sigma_{k}, \quad \mathbf{p}_{t}(\mathbf{x})=\left[H_{t}(\mathbf{x})\right] \mathbf{p}_{t}, \quad \mathbf{u}_{k}(\mathbf{x})=\left[H_{u k}(\mathbf{x})\right] \mathbf{u}_{k}, \\
& \lambda_{k}(\mathbf{x})=\sum_{i=1}^{\xi}\left[H_{\lambda k i}(\mathbf{x})\right] \lambda_{k i}=\left[H_{\lambda k}(\mathbf{x})\right] \lambda_{k}, \quad \lambda_{t}(\mathbf{x})=\left[H_{\lambda t}(\mathbf{x})\right] \lambda_{t}, \quad C_{k}(\mathbf{x})=\left[H_{0 k}(\mathbf{x})\right] \mathbf{C}_{k} .
\end{aligned}
$$


Здесь $\sigma_{k}, \mathbf{u}_{k}, \mathbf{C}_{k}, \lambda_{k}-$ векторн напряхений, перемещений, параметров текучести и пластических множителей узловнх точек $k$-го элемента. Матрицы фунапий формы $\left[H_{u k}(\mathbf{x})\right],\left[H_{k}(\mathbf{x})\right]$ и векторы $\mathbf{u}_{k}, \sigma_{k}$ обобщенных перемещений и напряжений дољжны бнть построены тақ, чтобы при равенстве узловьх перемещений и напряжений смежншх конечнбх элементов соблюдалась неразрнвность функпий перемещений и напряжений и другие требования сходимости [11].

Смешанные постановки задачи. После подстанонки функщи (5),(6) задача (2) получает следуюший дискретншй вид:

$$
\begin{aligned}
& \mathscr{E}_{1}=\sum_{t \in \mathscr{O}_{,}}\left(\mathbf{u}_{t}^{-}\right)^{T} \mathbf{F}_{t}+\sum_{k} \frac{1}{2} \sigma_{k}^{T}\left[D_{k}\right] \sigma_{k}+\sum_{k} \sigma_{k}^{T} \varepsilon_{0 k}-\sum_{k} \sigma_{k}^{T}\left[A_{k}\right]^{T} \mathbf{u}_{k}+\sum_{t \in \mathscr{G}_{0}} \mathbf{p}_{t}^{T}\left[A_{\mathbf{v} t}\right]^{T}\left(\mathbf{u}_{t}^{+}-\mathbf{u}_{t}^{-}\right)+ \\
& +\sum_{t \in \mathscr{T} .} \mathbf{p}_{t}^{T}\left[A_{u t}\right]^{T}\left(\mathbf{u}_{t}^{+}-\mathbf{u}_{0 t}\right)-\sum_{k} \lambda_{k}^{T}\left\{\mathbf{f}_{0}\left(\mathbf{C}_{k}\right)-\mathbf{f}_{k}\left(\sigma_{k}\right)\right\}-\sum_{t \in \mathscr{F}^{\mathscr{T}}} \lambda_{t}^{T}\left\{\mathbf{f}_{0 t}\left(\mathbf{C}_{t}\right)-\mathbf{f}_{t}\left(\mathbf{p}_{t}\right)\right\} \rightarrow \text { stac }
\end{aligned}
$$

при условиях

$$
\lambda_{k} \geq 0, \quad k \in \mathscr{K} ; \quad \lambda_{t} \geq \mathbf{0}, \quad t \in \mathscr{G} .
$$

Здесь вехторы внешних узловьх сил, взвешенншх началынхх деформаций

$$
\mathbf{F}_{t}=\int_{S_{f}}\left[H_{u t}(\mathbf{x})\right]^{T} \mathbf{F}_{t}(\mathbf{x}) d S_{t}, \quad \varepsilon_{0 k}=\int_{V_{k}}\left[H_{k}(\mathbf{x})\right]^{T} \varepsilon_{0 k}(\mathbf{x}) d V_{k}
$$

и фунхай текучести хонечного элемента

$$
\begin{array}{ll}
\mathbf{f}_{k}\left(\sigma_{k}\right)=\int_{V_{k}}\left[H_{\lambda k}(\mathbf{x})\right]^{T} \mathbf{f}\left(\sigma_{k}(\mathbf{x})\right) d V_{k}, & \mathbf{f}_{0 k}\left(\mathbf{C}_{k}\right)=\int_{V_{k}}\left[H_{\lambda k}(\mathbf{x})\right]^{T} \mathbf{f}_{0}\left(C_{k}(\mathbf{x})\right) d V_{k}, \\
\mathbf{f}_{t}\left(\mathbf{p}_{t}\right)=\int_{S_{t}}\left[H_{\lambda t}(\mathbf{x})\right]^{T} \mathbf{f}_{t}\left(\mathbf{p}_{t}(\mathbf{x})\right) d S_{t}, & \mathbf{f}_{0 t}\left(\mathbf{C}_{t}\right)=\int_{S_{t}}\left[H_{\lambda_{t}}(\mathbf{x})\right]^{T} \mathbf{f}_{0 t}\left(C_{t}(\mathbf{x})\right) d S_{t} .
\end{array}
$$

Матрища податливости элемента

$$
\left[D_{k}\right]=\int_{V_{k}}\left[H_{k}(\mathbf{x})\right]^{T}\left[d_{k}\right]\left[H_{k}(\mathbf{x})\right] d V_{k}
$$

и матришы

$$
\left[A_{k}\right]^{T}=\int_{V_{k}}\left[H_{k}(\mathbf{x})\right]^{T}[\mathscr{A}]^{T}\left[H_{u k}(\mathbf{x})\right] d V_{k}, \quad\left[A_{\mathbf{v t}}\right]^{T}=\int_{S_{t}}\left[H_{t}(\mathbf{x})\right]^{T}\left[H_{u t}(\mathbf{x})\right] d S_{t}
$$

Условие стационарности функционала геометрические уравнения конечных элементов и условия совместности перемещений между конечнбти элементами и на граничной поверхности тела. Принимая их за предварительные условия, задачу определения сташионарной точки функционала $\mathscr{F}_{1}$ преобразуем в задачу минимизации полной энергии:

$$
\mathbf{u}^{T} \mathbf{F}-\frac{1}{2} \sigma^{T}[D] \sigma-\lambda^{T}[\nabla \mathbf{f}(\sigma)] \sigma-\lambda_{p}^{T}\left[\nabla \mathbf{f}_{p}(\mathbf{p})\right] \mathbf{p}-\lambda^{T}\left\{\mathbf{f}_{0}(\mathbf{C})-\mathbf{f}(\sigma)\right\}-\lambda_{p}^{T}\left\{\mathbf{f}_{0_{p}}(\mathbf{C})-\mathbf{f}_{p}(\mathbf{p})\right\} \rightarrow \max
$$

при условиях

$$
\begin{array}{rr}
{[D] \sigma+[\nabla \mathbf{f}(\sigma)]^{T} \lambda-[A]^{T} \mathbf{u}=-\boldsymbol{\varepsilon}_{0},} & \lambda \geq \mathbf{0} \\
{\left[\nabla \mathbf{f}_{\mathbf{v}}\left(\mathbf{p}_{\mathbf{v}}\right)\right]^{T} \lambda_{\mathbf{v}}-\left[A_{\mathbf{v}}\right]^{T} \mathbf{u}=\mathbf{0},} & \lambda_{\mathbf{v}} \geq \mathbf{0} \\
{\left[\nabla \mathbf{f}_{u}\left(\mathbf{p}_{u}\right)\right]^{T} \lambda_{u}+\left[A_{u}\right]^{T} \mathbf{u}=\left[A_{u}\right]^{T} \mathbf{u}_{0},} & \lambda_{u} \geq \mathbf{0} .
\end{array}
$$


3десь $[D],[A],[\nabla \mathbf{f}(\sigma)]-$ алтебраическис матрицы, диагональными блоками которых являются матришы $\left[D_{k}\right],\left[A_{k}\right]$ и $\left[\nabla \mathbf{f}_{k}\left(\sigma_{k}\right)\right]$ соответственно. Векторы ш, $\mathbf{F}, \sigma, \varepsilon_{0}$ составияет совокупность векторов $\mathbf{u}_{k}, \mathbf{F}_{k}, \sigma_{k}$ и $\varepsilon_{0 k}, k=1,2, \ldots, s$. Составляюццими векторов $\lambda_{p} \equiv\left\{\lambda_{v}, \lambda_{u}\right\}^{T}$ и $\mathbf{p} \equiv\left\{\mathbf{p}_{v}, \mathbf{p}_{u}\right\}^{T}$ являются векторы пластических множителей $\lambda_{t}$ и напряжений $\mathbf{p}_{t}$ всех поверхностей конечных элементов.

Если начальные перемсщения отсутствуют, т. е. $\mathbf{u}_{0}=\mathbf{0}$, то кинематические траничные условия удобно учитывать исключением нулевых составляющих вектора перемещений и соответствующих столбцов матриц геометрически уравнений. Тогда уравнения совместности перемещений можно объединить. В этом случае получаем следующую математическую модель задачи:

$$
\mathbf{u}^{T} \mathbf{F}-\frac{1}{2} \sigma^{T}[D] \sigma-\lambda^{T}[\nabla \mathbf{f}(\sigma)] \sigma-\lambda_{p}^{T}\left[\nabla \mathbf{f}_{p}(\mathbf{p})\right] \mathbf{p}-\lambda^{T}\left\{\mathbf{f}_{0}(\mathbf{C})-\mathbf{f}(\sigma)\right\}-\lambda_{p}^{T}\left\{\mathbf{f}_{0 p}(\mathbf{C})-\mathbf{f}_{p}(\mathbf{p})\right\} \rightarrow \max
$$

при условиях

$$
\begin{aligned}
{[D] \boldsymbol{\sigma}+\mathbf{\varepsilon}_{0}+[\nabla \mathbf{f}(\sigma)]^{T} \lambda-[A]^{T} \mathbf{u}=\mathbf{0}, } & \lambda \geq \mathbf{0} \\
{\left[\nabla \mathbf{f}_{p}(\mathbf{p})\right]^{T} \lambda_{p}-\left[A_{p}\right]^{T} \mathbf{u}=\mathbf{0}, } & \lambda_{p} \geq \mathbf{0} .
\end{aligned}
$$

Неизвестными в задачах (12) и (13) являотся векторы напряхений, перемещений и пластичесхих множителей. Как известно, трудоемкость и время, необходимое для решения задачи математического программирования, в первую очередь зависят от числа неизвестных. Поэтому всегда необходимо стремиться уменышить число неизвестных задачи. Если геометрические уравнения конечных элементов линейны, то напряжения можно внразить через деформации и искпочить из числа неизвестнбх. Тахой случай рассматривается в пятом разделе статыи. Если хе эти уравнения нелинейны и необратимь, то такая возможность не существует. Однако и в этом случае можно уменыпить тисло неизвестных задачи путем искюочения перемещений.

Систему ограничений полной дополнительной энергии в задачах (12) и (13) можно представить одним матричным уравнением

где

$$
[\tilde{D}] \sigma+[\nabla \tilde{\mathbf{f}}(\sigma, \mathbf{p})]^{T} \tilde{\lambda}-[\tilde{A}]^{T} \mathbf{u}=\tilde{\varepsilon_{0}}, \quad \tilde{\lambda} \geq \mathbf{0},
$$

$$
[\widetilde{D}]=\left[\begin{array}{c}
{[D]} \\
{[0]} \\
{[0]}
\end{array}\right], \quad[\widetilde{A}]^{T}=\left[\begin{array}{c}
{[A]^{T}} \\
{\left[A_{\mathbf{v}}\right]^{T}} \\
-\left[A_{u}\right]^{T}
\end{array}\right], \quad \widetilde{\varepsilon}_{0}=\left|\begin{array}{c}
-\varepsilon_{0} \\
\mathbf{0} \\
{\left[A_{u}\right]^{T} \mathbf{u}_{0}}
\end{array}\right|, \quad[\nabla \tilde{\mathbf{f}}(\sigma, \mathbf{p})]=\left[\begin{array}{r}
{[\nabla \mathbf{f}(\sigma)]} \\
{\left[\nabla \mathbf{f}_{\mathbf{v}}\left(\mathbf{p}_{\mathbf{v}}\right)\right]} \\
{\left[\nabla \mathbf{f}_{u}\left(\mathbf{p}_{u}\right)\right]}
\end{array}\right] .
$$

Вектор $\tilde{\lambda}=\left\{\lambda, \lambda_{v}, \lambda_{u}\right\}^{T}$. Вибирая базисную матрицу $\left[\widetilde{A}_{1}\right]^{T}$ и разделяя все матрицы и вектор $\widetilde{\varepsilon}_{0}$ уравнения (14) на две части, получаем решение

$$
\mathbf{u}=\left(\left[\tilde{A}_{1}\right]^{T}\right)^{-1}\left\{\left[\widetilde{D}_{1}\right] \sigma+[\nabla \tilde{\mathbf{q}}(\sigma, \mathbf{p})]^{T} \tilde{\lambda}-\widetilde{\varepsilon}_{01}\right\}
$$

и уравнение совместности деформаций

Здесь

$$
\left[B_{\mathbf{\sigma}}\right] \sigma+\left[B_{\lambda}(\sigma, \mathbf{p})\right] \tilde{\lambda}=\Theta_{0}, \quad \tilde{\lambda} \geq \mathbf{0} .
$$




$$
\begin{aligned}
& {\left[B_{\mathbf{\sigma}}\right]=\left[\widetilde{D}_{2}\right]-\left[\widetilde{A}_{2}\right]^{T}\left(\left[\widetilde{A}_{1}\right]^{T}\right)^{-1}\left[\widetilde{D}_{1}\right], \quad \Theta_{0}=\widetilde{\varepsilon}_{02}-\left[\widetilde{A}_{2}\right]^{T}\left(\left[\widetilde{A}_{1}\right]^{T}\right)^{-1} \widetilde{\varepsilon}_{01},} \\
& {\left[B_{\lambda}(\sigma, \mathbf{p})\right]=\left[\nabla \widetilde{\mathbf{f}}_{2}(\sigma, \mathbf{p})\right]^{T}-\left[\widetilde{A}_{2}\right]^{T}\left(\left[\widetilde{A}_{1}\right]^{T}\right)^{-1}\left[\nabla \tilde{\mathbf{f}}_{1}(\sigma, \mathbf{p})\right]^{T} .}
\end{aligned}
$$

Зависимость (15) позволяет исключить перемещечия из тисла неизвестных. Получается модифищированная математическая модель смешанной постановки задачи:

$$
\begin{aligned}
& \mathbf{F}^{T}\left(\left[\widetilde{A}_{1}\right]^{T}\right)^{-1}\left\{\left[\widetilde{D}_{1}\right] \sigma+[\nabla \tilde{\mathbf{f}}(\sigma, \mathbf{p})]^{T} \tilde{\lambda}-\widetilde{\varepsilon}_{01}\right\}-\frac{1}{2} \sigma^{T}[D] \sigma-\lambda^{T}[\nabla \mathbf{f}(\sigma)] \sigma-\lambda_{p}^{T}\left[\nabla \mathbf{f}_{p}(\mathbf{p})\right] \mathbf{p}- \\
& -\lambda^{T}\left\{\mathbf{f}_{0}(\mathbf{C})-\mathbf{f}(\sigma)\right\}-\lambda_{p}^{T}\left\{\mathbf{f}_{0_{p}}(\mathbf{C})-\mathbf{f}_{p}(\mathbf{p})\right\} \rightarrow \max
\end{aligned}
$$

при условият (16).

Статические постановки задачи. Методом множителей Лагранжа смешанные постановхи (12) и (13) могут быть преобразованы в двойственшые $\mathbf{x}$ ним статические постановхи задачи. Математическая модель статической постановхи при наличии начальных перемещений имеет вид:

$$
\frac{1}{2} \sigma^{T}[D] \sigma+\sigma^{T} \varepsilon_{0}-\mathbf{p}_{u}^{T}\left[A_{u}\right]^{T} \mathbf{u}_{0} \rightarrow \min
$$

при условият

$$
\mathbf{f}_{0}(\mathbf{C})-\mathbf{I}(\sigma) \geq \mathbf{0}, \quad \mathbf{f}_{0 p}(\mathbf{C})-\mathbf{f}_{p}(\mathbf{p}) \geq \mathbf{0}, \quad[A] \sigma+\left[A_{\mathbf{v}}\right] \mathbf{p}_{\mathbf{v}}-\left[A_{u}\right] \mathbf{p}_{u}=\mathbf{F}
$$

Фуниция цели этой задачи вырахает полную дополнительную энергию дискретного тела. Неравенства представляот собой условия текучести конечнбх элементов и мехэлементнбх поверхностей, а равенства - уравнения равновесия конечноэлементной модели. Поэтому задача (19) отвечает минималыному принципу дополнительной энергии и позволяет определить нихпие значения напряжений в конечнбх элементах, в том числе и на их поверхностях.

Математическая модель статичесхой постановки задачи при отсутствии начальнбх перемещений:

$$
\frac{1}{2} \sigma^{T}[D] \sigma+\sigma^{T} \varepsilon_{0} \rightarrow \min
$$

при условиях

$$
\mathbf{f}_{0}(\mathbf{C})-\mathbf{f}(\boldsymbol{\sigma}) \geq \mathbf{0}, \quad \mathbf{f}_{0 p}(\mathbf{C})-\mathbf{f}_{p}(\mathbf{p}) \geq \mathbf{0}, \quad[A] \boldsymbol{\sigma}+\left[A_{p}\right] \mathbf{p}=\mathbf{F}
$$

Математичесхие модели задачи при отсутствии начаљынх деформащй получаются исключением вектора начальньх деформаций.

\section{2. Задача анализа остаточных перемещений и напряжений}

В тех случаях, когда конструхпия подвергается действию разных воздействий или упругие параметры НДС известни или нетрудно могут быть определены, задачу упругопластическопо анализа конструхапи удобно формулировать в терминах остаточных напряжений и перемещений. Дискретные математические модели задачи анализа остаточных параметров НДС строятся аналогично - путем дискретизации задачи (4).

Для хаждого конечного элемента принимаются апптроксимирующие функпии (6) и

$$
\sigma_{r k}(\mathbf{x})=\sum_{i=1}^{\xi}\left[H_{k i}(\mathbf{x})\right] \sigma_{r k i}=\left[H_{k}(\mathbf{x})\right] \sigma_{r k}, \quad \mathbf{p}_{r t}(\mathbf{x})=\left[H_{t}(\mathbf{x})\right] \mathbf{p}_{r t}, \quad \mathbf{u}_{r k}(\mathbf{x})=\left[H_{u k}(\mathbf{x})\right] \mathbf{u}_{r k} .
$$


Подставляя эти фунхции в функционал задачи (4) и используя вышеописанную методику, охончательно получаем следуюшую пару конечноэлементных математических моделей этой задачи:

а) в смешанной постановке -

$$
\begin{aligned}
& -\frac{1}{2} \sigma_{r}^{T}[D] \sigma_{r}-\lambda^{T}\left[\nabla \mathbf{f}\left(\sigma_{e}+\sigma_{r}\right)\right] \sigma_{r}-\lambda_{p}^{T}\left[\nabla \mathbf{f}_{p}\left(\mathbf{p}_{e}+\mathbf{p}_{r}\right)\right] \mathbf{p}_{r}-\lambda^{T}\left\{\mathbf{f}_{0}(\mathbf{C})-\mathbf{f}\left(\sigma_{e}+\sigma_{r}\right)\right\}- \\
& -\lambda_{p}^{T}\left\{\mathbf{f}_{0 p}(\mathbf{C})-\mathbf{f}_{p}\left(\mathbf{p}_{e}+\mathbf{p}_{r}\right)\right\} \rightarrow \max
\end{aligned}
$$

при условиях

$$
\begin{array}{rr}
{[D] \sigma_{r}+\left[\nabla \mathbf{f}\left(\sigma_{e}+\sigma_{r}\right)\right]^{T} \lambda-[A]^{T} \mathbf{u}_{r}=\mathbf{0},} & \lambda \geq \mathbf{0} ; \\
{\left[\nabla \mathbf{f}_{p}\left(\mathbf{p}_{e}+\mathbf{p}_{r}\right)\right]^{T} \lambda_{p}-\left[A_{p}\right]^{T} \mathbf{u}_{r}=\mathbf{0},} & \lambda_{p} \geq \mathbf{0} ;
\end{array}
$$

б) в статической постановке -

$$
\frac{1}{2} \sigma_{r}^{T}[D] \sigma_{r} \rightarrow \min
$$

при условиях

$$
\mathbf{f}_{0}(\mathbf{C})-\mathbf{I}\left(\sigma_{e}+\sigma_{r}\right) \geq \mathbf{0}, \quad \mathbf{f}_{0 p}(\mathbf{C})-\mathbf{f}_{p}\left(\mathbf{p}_{e}+\mathbf{p}_{r}\right) \geq \mathbf{0}, \quad[A] \sigma_{r}+\left[A_{p}\right] \mathbf{p}=\mathbf{0} .
$$

Она выражает пару двойственньх экстремальннх приниипов о минимуме полной энергии остаточных деформаций и о минимуме дополнительной энергии остаточных напряжений для конечноэлементной модели тела и позволяет определить нижние оценки остаточных перемещений и натряжений.

Модифипировантая математическая модель смешанной постановхи задачи:

$$
\begin{aligned}
& -\frac{1}{2} \sigma_{r}^{T}[D] \sigma_{r}-\lambda^{T}\left[\nabla \mathbf{f}\left(\sigma_{e}+\sigma_{r}\right)\right] \sigma_{r}-\lambda_{p}^{T}\left[\nabla \mathbf{f}_{p}\left(\mathbf{p}_{e}+\mathbf{p}_{r}\right)\right] \mathbf{p}_{r}-\lambda^{T}\left\{\mathbf{f}_{0}(\mathbf{C})-\mathbf{f}\left(\sigma_{e}+\sigma_{r}\right)\right\}- \\
& -\lambda_{p}^{T}\left\{\mathbf{f}_{0 p}(\mathbf{C})-\mathbf{f}_{p}\left(\mathbf{p}_{e}+\mathbf{p}_{r}\right)\right\} \rightarrow \max
\end{aligned}
$$

при условиях

$$
\left[B_{\sigma}\right] \sigma_{r}+\left[B_{\lambda}(\sigma, \mathbf{p})\right] \tilde{\lambda}=\mathbf{0}, \quad \tilde{\lambda} \geq \mathbf{0}
$$

Построенные нами формальньм путем математические модели (19) и (22) статической постановқи задач анализа НДС ни по виду, ни по существу не отличаются от представленнвбх в [3] статических постановок этих задач, построенных с применением равновеснкх конечных элементов. Разница состоит в происхождении матрищ коэффиџиентов уравнений равновесия. В работе [3] они построены на основе апгроксимаций уравнений равновесия сплошното тела и являотся равновеснбми, а в данной работе они построены на основе аптроксимаций полей перемещений и напряжений. Поэтому все вышеприведенные математичесхие модели, в том числе и математичесхие модели статической постановки задач, принадлежат классу смешаншх постановок.

\section{4. Аналнз условнй текучести в геометрнческих уравнений}

Выражения $\varphi_{k}=\mathbf{f}_{0 k}\left(\mathbf{C}_{k}\right)-\mathbf{f}_{k}\left(\sigma_{k}\right) \geq \mathbf{0}, \quad \boldsymbol{\varphi}_{t}=\mathbf{f}_{0 t}\left(\mathbf{C}_{t}\right)-\mathbf{f}_{t}\left(\mathbf{p}_{t}\right) \geq \mathbf{0}, \quad$ входяшие в математические модели задач, представляют собой дискретные условия техучести $\boldsymbol{k}$-го элемента и $\boldsymbol{t}$-й поверхности раздела элементов. Все составляюпие этих условий огределены зависимостями (9). Из них следует, что выбор разных аппроксимируюших функий пластических множителей приводит к разнвм выражениям дискретных условий текучести, соответствуюцих разным методам коллокаций $[3,12]$. 
Геометрические уравнения (14) конечноэлементной модели конструкщии получены как условия стапионарности дискретно описанного фунхпионала . Однако возможюк и другие способы учета и дискретизации теометрических уравнений. Если уравнения (3) линейны, то напряжения можно выразить через деформации и исключить из числа неизвестных задачи. В этом случае геометрические уравнения соблюдаются во всех точках конечного элемента. Если же геометрические уравнения конечных элементов нелинейны и необратимs, то они могут быть удовлетворены только в слабой форме:

$$
\int_{V_{k}}\left[G_{k}(\mathbf{x})\right]^{T}\left\{\left[d_{k}\right] \sigma_{k}(\mathbf{x})+\varepsilon_{0 k}(\mathbf{x})+\left[\nabla \mathbf{f}\left(\sigma_{k}(\mathbf{x})\right)\right]^{T} \lambda_{k}(\mathbf{x})-[\mathscr{C A}]^{T} \mathbf{u}_{k}(\mathbf{x})\right\} d V_{k}=\mathbf{0},
$$

где $\left[G_{k}(\mathbf{x})\right]$ - матрища весовьх функций, зависящая от используемого метода коллокаший. Для метода коллокации в точке и метода Бубнова-Галеркина она состоит из подматрищ $\left[G_{k i}(\mathbf{x})\right]$, соответствуюших узлам $i=1,2, \ldots, \xi$. После интегрирования получаются алгебраические уравнения совместности конечного элемента:

$$
\left[\bar{D}_{k}\right] \sigma_{k}+\bar{\varepsilon}_{0 k}+\varepsilon_{p k}-\left[\bar{A}_{k}\right]^{T} \mathbf{u}_{k}=\mathbf{0}
$$

Выбор разных весовых функшй, соответствуюших разнжм методам коллохаций, приведет к разным выражениям матрищ $\left[\bar{D}_{k}\right],\left[\bar{A}_{k}\right]$ и векторов начальньх и пластических деформаций элемента. Согласно методу коллохации в точхе $\left[G_{k i}\left(\mathbf{x}_{i}\right)\right]=[E]$ для узловой точхи $i$ и $\left[G_{k i}\left(\mathbf{x}_{j}\right)\right]=[0]$ для всех остальных точек элемента. Тогда $\left[\bar{D}_{k}\right]=\operatorname{diag}\left[d_{k}\right], \varepsilon_{p k}=\left\{\varepsilon_{p k 1}, \varepsilon_{p k 2}, \ldots, \varepsilon_{p k \xi}\right\}^{T}$, где $\varepsilon_{p k i}=\left[\nabla \mathbf{f}_{k i}\left(\sigma_{k i}\right)\right]^{T} \lambda_{k i}$, $\bar{\varepsilon}_{0 k} \equiv\left\{\varepsilon_{0 k i}\right\}$. Матрица $\left[\vec{A}_{k}\right]$ состоит из подматриц $\left[A_{k i}\right]^{T}=\left[A_{k}\left(\mathbf{x}_{i}\right)\right]^{T}$, где $\left[A_{k}(\mathbf{x})\right]=[\mathscr{A}]^{T}\left[H_{u k}(\mathbf{x})\right]$. В этом случае геометрические уравнения элемента (25) вырахаются совокупностью уравнений

$$
\left[d_{k}\right] \sigma_{k i}+\varepsilon_{0 k i}+\left[\nabla \mathbf{f}_{k i}\left(\sigma_{k i}\right)\right]^{T} \lambda_{k i}-\left[A_{k i}\right]^{T} \mathbf{u}_{k}=\mathbf{0}, \quad i=1,2, \ldots, \xi
$$

и удовлетворяются лишь в узлах элемента. Используя метод холлокации в подобласти, полагаем $\left[G_{k}(\mathbf{x})\right]=[E]$. Тогда

$$
\begin{aligned}
& {\left[\bar{D}_{k}\right]=\int_{V_{k}}\left[d_{k}\right]\left[H_{k}(\mathbf{x})\right] d V_{k}, \quad\left[\bar{A}_{k}\right]=\int_{V_{k}}[\mathscr{\mathscr { A }}]^{T}\left[H_{u k}(\mathbf{x})\right] d V_{k},} \\
& \bar{\varepsilon}_{0 k}=\int_{V_{k}} \varepsilon_{0 k}(\mathbf{x}) d V_{k}, \quad \varepsilon_{p k}=\int_{V_{k}}\left[\nabla \mathbf{f}\left(\sigma_{k}(\mathbf{x})\right)\right]^{T}\left[H_{\lambda k}(\mathbf{x})\right] \lambda_{k} d V_{k} .
\end{aligned}
$$

Нетрудно заметить, что геометрические уравнения (25), полученные с использванием двух методов коллокаций, отличаются от уравнений (14), построеншт соответствующим методом. Лишь метод коллокации Бубнова-Галеркина с использованием весовьх матриц $\left[G_{k}(\mathbf{x})\right]=\left[H_{k}(\mathbf{x})\right]=\left[H_{\lambda k}(\mathbf{x})\right]$ приводит к геометрическим уравнениям, совпадающим с (14). Таким образом он дает наилучшую аптрокцимацию геометрических уравнений.

\section{5. Кинематнческие постановки зддач в случае линейных условий текучести}

В случае линейных условий текучести $\left[\Phi_{x}\right] \sigma_{k}(\mathbf{x}) \leq[\rho] \mathbf{C}_{k}(\mathbf{x}) \in V_{k}, \quad\left[\Phi_{b x}\right] \mathbf{p}_{t}(\mathbf{x}) \leq\left[\rho_{t}\right] \mathbf{C}_{t}(\mathbf{x}) \in S_{t}$ геометрические уравнения конетных элементов линейны, а $\left[\nabla \mathbf{f}\left(\sigma_{k}(\mathbf{x})\right)\right]=\left[\Phi_{x}\right],\left[\mathbf{f}_{t}\left(\mathbf{p}_{t}(\mathbf{x})\right)\right]=\left[\Phi_{t x}\right]$. Поэтому из математических моделей (2) и (4) можно искпючить напряжения и для дискретизации задач применять конечные элементы в перемещениях (совместные). Подставляя выражение 


$$
\sigma_{k}(\mathbf{x})=\left[d_{k}\right]^{-1}\left\{[\mathscr{A}]^{T} \mathbf{u}_{k}(\mathbf{x})-\varepsilon_{0 k}(\mathbf{x})-\left[\Phi_{x}\right]^{T} \lambda_{k}(\mathbf{x})\right\}
$$

в функционал задачи (2) и принимая аппроксимируюшие функции перемещений, пластических множителей и параметров пластичности, после некоторнх преобразований получаем дискретную хинематическую постановку задачи определения полных перемещений:

$$
\mathbf{u}^{T}\left(\mathbf{F}+\mathbf{F}_{0}\right)-\frac{1}{2} \mathbf{u}^{T}[K] \mathbf{u}-\frac{1}{2} \lambda^{T}\left[K_{\lambda}\right] \lambda-\lambda^{T} \sigma_{0}+\lambda^{T}\left[K_{u \lambda}\right] \mathbf{u}-\lambda^{T}[V] \mathbf{C}-\lambda_{p}^{T}\left[V_{p}\right] \mathbf{C}_{p} \rightarrow \max
$$

при условиях

$$
\left[\Phi_{\mathbf{v}}\right]^{T} \lambda_{\mathbf{v}}-\left[A_{\mathbf{v}}\right]^{T} \mathbf{u}=\mathbf{0}, \quad\left[\Phi_{u}\right]^{T} \lambda_{u}-\left[A_{u}\right]^{T}\left(\mathbf{u}_{0}-\mathbf{u}\right)=\mathbf{0}, \quad \lambda_{\mathbf{v}} \geq \mathbf{0}, \quad \lambda_{u} \geq \mathbf{0}
$$

Диатональньми блохами квазидиагональных матриц $[K],\left[K_{\lambda}\right],\left[K_{u \lambda}\right],[V],\left[V_{p}\right],\left[\Phi_{v}\right] \quad$ являются матришы

$$
\begin{aligned}
& {\left[k_{k}\right]=\int_{V_{k}}\left\{[\mathscr{A}]^{T}\left[H_{u k}(\mathbf{x})\right]\right\}^{T}\left[d_{k}\right]^{-1}[\mathscr{A}]^{T}\left[H_{u k}(\mathbf{x})\right] d V_{k}, \quad\left[V_{k}\right]=\int_{V_{k}}\left[H_{\lambda k}(\mathbf{x})\right]^{T}[\rho]\left[H_{0 k}(\mathbf{x})\right] d V_{k},} \\
& {\left[k_{\lambda k}\right]=\int_{V_{k}}\left[H_{\lambda k}(\mathbf{x})\right]^{T}\left[\Phi_{x}\right]\left[d_{k}\right]^{-1}\left[\Phi_{x}\right]^{T}\left[H_{\lambda k}(\mathbf{x})\right] d V_{k}, \quad\left[V_{p k}\right]=\int_{S_{t}}\left[H_{\lambda t}(\mathbf{x})\right]^{T}\left[\rho_{t}\right]\left[H_{0 t}(\mathbf{x})\right] d S_{t},} \\
& {\left[k_{u k}\right]=\int_{V_{k}}\left[H_{\lambda k}(\mathbf{x})\right]^{T}\left[\Phi_{x}\right]\left[d_{k}\right]^{-1}[\mathscr{A}]^{T}\left[H_{u k}(\mathbf{x})\right] d V_{k}, \quad\left[\Phi_{\mathbf{v} t}\right]=\int_{S_{t}}\left[H_{\lambda t}(\mathbf{x})\right]^{T}\left[\Phi_{t x}\right]\left[H_{t}(\mathbf{x})\right] d S_{t} .}
\end{aligned}
$$

Здесь $\left[k_{k}\right]$ - общеизвестная матрища жестхости $k$-го элемента. Составлношими векторов внешших узловьх сил $\mathbf{F}, \mathbf{F}_{0}$ и напряхений $\sigma_{0}$ являотся векторы

$$
\begin{aligned}
& \left\{\mathbf{F}_{0 k}\right\}=\int_{V_{k}}\left\{[\mathscr{A}]^{T}\left[H_{u k}(\mathbf{x})\right]\right\}^{T}\left[d_{k}\right]^{-1} \varepsilon_{0 k}(\mathbf{x}) d V_{k}, \quad\left\{\mathbf{F}_{k}\right\}=\int_{S_{\beta_{k}}}\left[H_{u k}(\mathbf{x})\right]^{T} \mathbf{F}_{k}(\mathbf{x}) d S, \\
& \left\{\sigma_{0 k}\right\}=\int_{V_{k}}\left[H_{\lambda k}(\mathbf{x})\right]^{T}\left[\Phi_{x}\right]\left[d_{k}\right]^{-1} \varepsilon_{0 k}(\mathbf{x}) d V_{k} .
\end{aligned}
$$

Аналогичнкм образом, после исключения вектора остаточных напряхений

$$
\sigma_{r k}(\mathbf{x})=\left[d_{k}\right]^{-1}\left\{[\mathscr{C A}]^{T} \mathbf{u}_{r k}(\mathbf{x})-\left[\Phi_{x}\right]^{T} \lambda_{k}(\mathbf{x})\right\}
$$

и дискретизации смешанная постановка (4) задачи анализа остаточных перемещений и напряххений преобразуется в дискретную кинематическую постановку:

$$
-\frac{1}{2} \mathbf{u}_{r}^{T}[K] \mathbf{u}_{r}-\frac{1}{2} \lambda^{T}\left[K_{\lambda}\right] \lambda+\lambda^{T}\left[K_{u \lambda}\right] \mathbf{u}_{r}-\lambda^{T}\left\{[V] \mathbf{C}-[\Phi] \sigma_{e}\right\}-\lambda_{p}^{T}\left\{\left[V_{p}\right] \mathbf{C}_{p}-\left[\Phi_{p}\right] \mathbf{p}_{e}\right\} \rightarrow \max
$$

при условиях

$$
\left[\Phi_{p}\right]^{T} \lambda_{p}-\left[A_{p}\right]^{T} \mathbf{u}_{r}=\mathbf{0}, \quad \lambda_{p} \geq \mathbf{0}
$$

Диагональными блоками квазидиагональных матрищ $[\Phi]$ и $\left[\Phi_{P}\right]$ явцяотся матрищы

$$
\left[\Phi_{k}\right]=\int_{V_{k}}\left[H_{\lambda k}(\mathbf{x})\right]^{T}\left[\Phi_{x}\right]\left[H_{k}(\mathbf{x})\right] d V_{k}, \quad\left[\Phi_{t}\right]=\int_{S_{t}}\left[H_{\lambda t}(\mathbf{x})\right]^{T}\left[\Phi_{t x} \mathbf{I} H_{t}(\mathbf{x})\right] d S_{t}
$$

условий текучести конечных элементов и их поверхностей. Матрица $\left[\Phi_{p}\right]$ состоит из $\left[\Phi_{\mathbf{v}}\right]$ и $\left[\Phi_{u}\right]$.

Математические модели (27) и (29) позволяют определить векторы перемещений и пластических множителей, на основе которьх в дальнейшем могуг бнть огределены функции напряжений с использованием формул (26) и (28). Преимущество математических моделей (27) и 
(29) по сравнению с (12) и (21) состоит не только в том, что они содержат меныше неизвестных, но и в том, что геометрические уравнения соблюдаются во всех точках конечных элементов. В задачах (12), (13) и (21) они соблюдаются липь в слабой форме (за исключением стержневых систем).

В представленных кинематических постановках задач неизвестными являются векторн перемещений и пластических множителей. Однако, как показано в работе [3] для равновесных моделей, в случае линейных условий текучести смешанные постановки (12) и (21) могут быть преобразованы в кинематические постановки задачи, в хоторых неизвестными являотся лишь пластические множители. Например, добавив уравнения равновесия х ограничениям задачи (21), полуучмм систему уравнений:

$$
\begin{gathered}
{[A] \sigma_{r}+\left[A_{p}\right] \mathbf{p}_{r}=\mathbf{0}, \quad[D] \sigma_{r}+[\Phi]^{T} \lambda-[A]^{T} \mathbf{u}_{r}=\mathbf{0},} \\
{\left[\Phi_{P}\right]^{T} \lambda_{p}-\left[A_{p}\right]^{T} \mathbf{u}_{r}=\mathbf{0}, \quad \lambda \geq \mathbf{0}, \quad \lambda_{p} \geq \mathbf{0} .}
\end{gathered}
$$

Решая ее относительно векторов пластических множителей, получаем зависимости:

$$
\mathbf{u}_{r}=[H] \lambda+\left[H_{\mathbf{v}}\right] \lambda_{p}, \quad \sigma_{r}=[G] \lambda+\left[G_{\mathbf{v}}\right] \lambda_{p}, \quad \mathbf{p}_{r}=\left[G_{p}\right] \lambda+\left[G_{p \mathbf{v}}\right] \lambda_{p} .
$$

Здесь матришы

$$
\begin{gathered}
{[H]=\left\{[\bar{K}]^{-1}-[\bar{K}]^{-1}\left[A_{p}\right]\left[K_{p}\right]^{-1}\left[A_{p}\right]^{T}[\bar{K}]^{-1}\right\}[A][D]^{-1}[\Phi]^{T}, \quad\left[H_{\mathrm{v}}\right]=[\bar{K}]^{-1}\left[A_{p}\right]\left[K_{p}\right]^{-1}\left[\Phi_{p}\right]^{T} ;} \\
{[G]=[D]^{-1}[A]^{T}[H]-[D]^{-1}[\Phi]^{T}, \quad\left[G_{\mathrm{v}}\right]=[D]^{-1}[A]^{T}\left[H_{\mathrm{v}}\right]=[D]^{-1}[A]^{T}[\bar{K}]^{-1}\left[A_{p}\right]\left[K_{p}\right]^{-1}\left[\Phi_{p}\right]^{T} ;} \\
{\left[G_{p}\right]=\left[K_{p}\right]^{-1}\left[A_{p}\right]^{T}[\bar{K}]^{-1}[A][D]^{-1}[\Phi]^{T}, \quad\left[G_{p \mathrm{v}}\right]=-\left[K_{p}\right]^{-1}\left[\Phi_{p}\right]^{T},}
\end{gathered}
$$

где

$$
[\bar{K}]=[A][D]^{-1}[A]^{T}, \quad\left[K_{p}\right]=\left[A_{P}\right]^{T}[\bar{K}]^{-1}\left[A_{p}\right]
$$

Подстановкой решения (31) в функщионал задачи (21) экстремалыная задача с ограничениями сводится $\mathbf{X}$ задаче махсимизации тольхо с условиями неотрицательности пластических множителей:

$$
-\frac{1}{2} \lambda^{T}[T] \lambda-\frac{1}{2} \lambda_{p}^{T}\left[T_{p}\right] \lambda_{p}-\lambda^{T}\left[T_{\lambda}\right] \lambda_{p}-\lambda^{T}\left\{[V] \mathbf{C}-[\Phi] \sigma_{e}\right\}-\lambda_{p}^{T}\left\{\left[V_{p}\right] \mathbf{C}_{p}-\left[\Phi_{p}\right] \mathbf{p}_{e}\right\} \rightarrow \max
$$

при условиях

$$
\lambda \geq \mathbf{0}, \quad \lambda_{p} \geq \mathbf{0} \text {. }
$$

Матрицы

$$
[T]=[G]^{T}[D][G], \quad\left[T_{p}\right]=\left[G_{\mathbf{v}}\right]^{T}[D]\left[G_{\mathbf{v}}\right], \quad\left[T_{\lambda}\right]=[G]^{T}[D]\left[G_{\mathbf{v}}\right]
$$

Преимушество математической модели (32) по сравнению с моделью (29) в том, что она содержит меныше неизвестных. Однако имеет и недостатох - геометрические уравнения конечных элементов, за искпючением стержневых систем, соблюдаются только в слабом (интегральном) виде. С применением равновесншх конечных элементов в работе [3] построена равновесная кинематическая постановка задачи анализа остаточных параметров НДС. Модель (32) отличается от нее только тем, что является не равновесной, а геометрически совместной.

Аналогичным образом можно преобразовать и статические постановки задач. Например, добавив геометрические уравнения $\mathbf{k}$ ограничениям задачи (22), получим систему ограниченийравенств (30). Тогда с учетом ее решения (31) статическая постановка (22) задачи анализа остаточных напряжений вырражается через кинематические величины: 


$$
\frac{1}{2} \lambda^{T}[T] \lambda+\frac{1}{2} \lambda_{p}^{T}\left[T_{p}\right] \lambda_{p}+\lambda^{T}\left[T_{\lambda}\right] \lambda_{p} \rightarrow \min
$$

при условиях

$$
\begin{array}{ll}
-[\Phi]\left\{[G] \lambda+\left[G_{\mathbf{v}}\right] \lambda_{p}\right\} \geq[\Phi] \sigma_{e}-[V] \mathbf{C}, & \lambda \geq \mathbf{0}, \\
-\left[\Phi_{p}\right]\left\{\left[G_{p}\right] \lambda+\left[G_{p \mathbf{v}}\right] \lambda_{p}\right\} \geq\left[\Phi_{p}\right] \mathbf{p}_{e}-\left[V_{p}\right] \mathbf{C}_{p}, & \lambda \geq \mathbf{0}
\end{array}
$$

Число ограничений и неизвестнбх в этой задаче меныше по сравнению с задачей (22). Все-таки реализация кинематической постановки (32) проще.

Аналогичным образом могут быть преобразованы и математические модели эадачи огределения полншх перемещений и напряжений. Однако при этом получаются более сложные задачи. Поэтому для определения пластических множителей целесообразно решить задачу (32). Для расчета перемещений и напряжений далее используются формулы (31). Это свидетельствует о том, что формулировка задач с соблюдением двойственннх связей позволяет значительно упростить алторитм решения задач упругопластического анализа.

\section{6. Прпмер построения н прмменения смепанных в совместных конечных элементов}

Анализ НДС упругопластических конструхций с применением совместных конечных элементов иллострируется на примере расчета простой двухпролетной балхи постоянного сечения (рис. 1) под действием равномерно распределенной нагрузки интенсивностью $p=17 M_{0} / 54$, где $M_{0}-$ предельный момент ее поперечного сечения. Требуется определить распределение изгибаюших моментов и перемещений (прогибов) балхи. Интенсивность нагрузки $p=17 M_{0} / 54$ принята с тахим расчетом, чтобы: во-первнх, имели место пластические деформации и, во-вторнх, выполнялось условие $p \leq p_{0}$, где $p_{0}$ - интенсивность предельной нагрузки.

1)

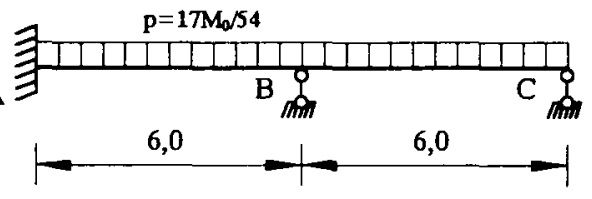

2)

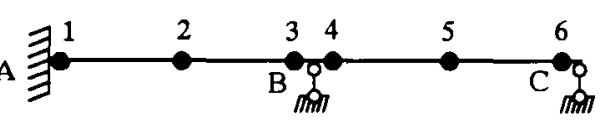

3)

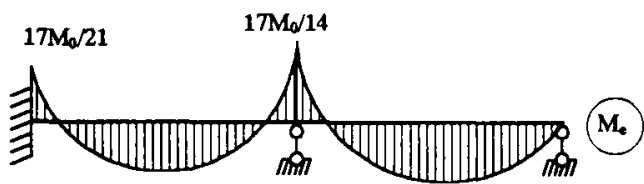

$3 \mathrm{M}_{0} / 28$

4)

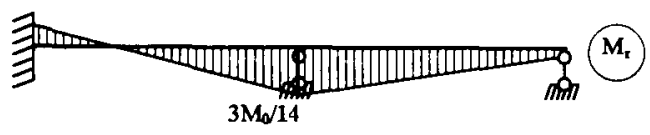

5)

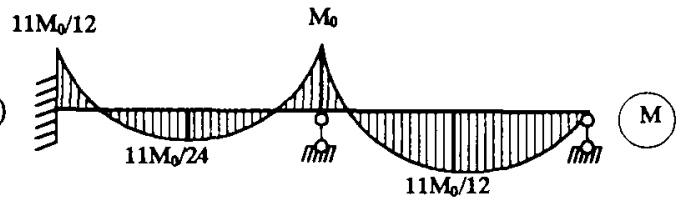

Рис. 1. Расчетная схема и результаты расчета балки
Поэтому вначале кинематическим методом предельного равновесия исследуется предельное состояние балки и огределяется величина предельной нагрузки $p_{0}=0,3238 M_{0} . \quad \mathrm{Ee}$ предельное состояние достигается при образовании двух пластических шарниров: над средней опорой и во втором пролете на расстоянии $x=3,515 \mathrm{~m}$ от средней опорн. Для решения задачи можно использовать широко известнй стержневой элемент $\mathrm{c}$ линейным распределением изгибающих моментов и кубическим распределением грогибов, содержашим четыре степени свободы. Однахо при этом необходимо делить каждый пролет балки на несколько конечных элементов. Так хак изгибающие моменты в балке распределяются по закону квадратичной параболы, целесообразнее построить элемент более высокого порядка.

Строим новый конечный элемент с параболическим распределением изгибающих моментов и функцией перемещений четвертой 


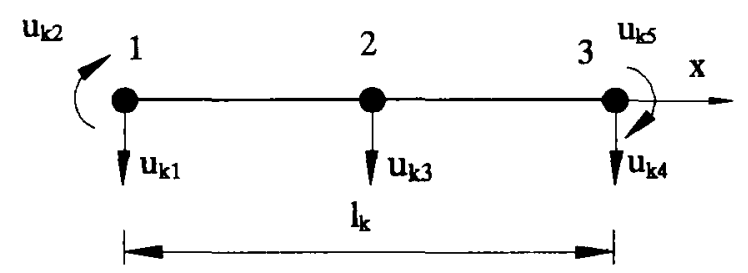

Рис. 2. Конечный элемент степени. Элемент имеет три узла и пять степеней свободы (рис. 2). Принимаются функции

$$
\begin{aligned}
& M_{k}(x)=a_{1}+a_{2} x+a_{3} x^{2}=\left\{\mathbf{h}_{k}(x)\right\}^{T} \mathbf{a}_{k}, \\
& \lambda_{k}(x)=b_{1}+b_{2} x+b_{3} x^{2}=\left\{\mathbf{h}_{k}(x)\right\}^{T} \mathbf{b}_{k},
\end{aligned}
$$

$$
u_{k}(x)=c_{1}+c_{2} x+c_{3} x^{2}+c_{4} x^{3}+c_{5} x^{4}=\left\{\mathbf{h}_{u k}(x)\right\}^{T} \mathbf{c}_{k} .
$$

С использованием граничных условий

$$
\begin{array}{lll}
M_{k i}=\left\{\mathbf{h}_{k}\left(x_{i}\right)\right\}^{T} \mathbf{a}_{k}, & i=1,2,3 ; & \lambda_{k i}=\left\{\mathbf{h}_{k}\left(x_{i}\right)\right\}^{T} \mathbf{b}_{k}, \quad i=1,2,3 ; \\
u_{k i}=\left\{\mathbf{h}_{u k}\left(x_{i}\right)\right\}^{T} \mathbf{c}_{k}, & i=1,3,4 ; & u_{k i}=\left[-\frac{\partial}{\partial x}\right]\left\{\mathbf{h}_{u k}\left(x_{i}\right)\right\}^{T} \mathbf{c}_{k}, \quad i=2,5
\end{array}
$$

неизвестные коэффищиенты $a_{i}, b_{i}, c_{i}$ соответствующих полиномов выражаются через узовые изгибающие моменты $\left\{\mathbf{M}_{k}\right\}=\left\{M_{k 1}, M_{k 2}, M_{k 3}\right\}^{T}$, обобщенные перемещения $\left\{\mathbf{u}_{k}\right\}=\left\{u_{k 1}, u_{k 2}, \ldots, u_{k 5}\right\}^{T}$ и пластические множители $\left\{\lambda_{k}\right\}=\left\{\lambda_{k 1}, \lambda_{k 2}, \lambda_{k 3}\right\}^{T}$, а аппроксимируютче функиии конечного элемента преобразуются к следуюшим выражениям:

$$
M_{k}(x)=\sum_{i=1}^{i=3} H_{k i}(x) M_{k i}=\left\{\mathbf{H}_{k}(x)\right\}^{T} \mathbf{M}_{k}, \quad u_{k}(x)=\sum_{i=1}^{i=5} H_{u k i}(x) u_{k i}=\left\{\mathbf{H}_{u k}(x)\right\}^{T} \mathbf{u}_{k}, \quad \lambda_{k}(x)=\left\{\mathbf{H}_{k}(x)\right\}^{T} \lambda_{k} .
$$

Здесь функщии формы

$$
\begin{aligned}
& H_{k 1}(x)=1-\frac{3 x}{l_{k}}+\frac{2 x^{2}}{l_{k}^{2}}, \quad H_{k 2}(x)=\frac{4 x}{l_{k}}-\frac{4 x^{2}}{l_{k}^{2}}, \quad H_{k 3}(x)=-\frac{x}{l_{k}}+\frac{2 x^{2}}{l_{k}^{2}} ; \\
& H_{u k 1}(x)=1-\frac{11 x^{2}}{l_{k}^{2}}+\frac{18 x^{3}}{l_{k}^{3}}-\frac{8 x^{4}}{l_{k}^{4}}, \quad H_{u k 2}(x)=x-\frac{4 x^{2}}{l_{k}}+\frac{5 x^{3}}{l_{k}^{2}}-\frac{2 x^{4}}{l_{k}^{3}}, \\
& H_{u k 3}(x)=\frac{16 x^{2}}{l_{k}^{2}}-\frac{32 x^{3}}{l_{k}^{3}}+\frac{16 x^{4}}{l_{k}^{4}}, \quad H_{u k 4}(x)=-\frac{5 x^{2}}{l_{k}^{2}}+\frac{14 x^{3}}{l_{k}^{3}}-\frac{8 x^{4}}{l_{k}^{4}}, \quad H_{u k 5}(x)=\frac{x^{2}}{l_{k}}-\frac{3 x^{3}}{l_{k}^{2}}+\frac{2 x^{4}}{l_{k}^{3}} .
\end{aligned}
$$

Дискретная модель балки строится разделением ее на два конечных элемента, соединеннњх в узле $B$ (рис 1.2). Согласно граничным условиям вертикаљьне перемещения узлов $i=1,3,4,6$ равны нуло и изгибаюший момент $M_{C}=0$. Поэтому неизвестными задачи являотся вехторы перемещений $\mathbf{u}=\left\{u_{12}, u_{13}, u_{15}, u_{22}, u_{23}, u_{25}\right\}^{T}$, изгибаюших моментов $\{\sigma\} \equiv\{\mathbf{M}\}=\left\{M_{1}, M_{2}, \ldots, M_{6}\right\}^{T}$ и $\mathbf{p}=\left\{M_{a}, M_{b}\right\}^{T}$ и пластических множителей $\lambda=\left\{\lambda_{1}, \lambda_{2}, \ldots, \lambda_{6}\right\}^{T}, \lambda_{p}=\left\{\lambda_{a}, \lambda_{b}\right\}^{T}$. Решение задачи можно определить согласно любой внше представленной математической модели.

Рассмотрим условия текучести, геометрические уравнения $\boldsymbol{k}$-го элемента и дискретной модели балки. Интегральное условие текучести конечного элемента выражается неравенством

$$
\left[\Phi_{k}\right] \mathbf{M}_{k}=\frac{l_{k}}{15}\left[\begin{array}{ccc}
2 & 1 & -0,5 \\
1 & 8 & 1 \\
-0,5 & 1 & 2
\end{array}\right] \times\left|\begin{array}{c}
M_{k 1} \\
M_{k 2} \\
M_{k 3}
\end{array}\right| \leq \frac{M_{0 k} l_{k}}{6}\left|\begin{array}{c}
1 \\
4 \\
1
\end{array}\right|=\mathbf{C}_{k} .
$$

Для узлов $A$ и $B$ должны соблюдаться условия текучести $-M_{a} \leq M_{0}$ и $-M_{b} \leq M_{0}$. Геометрическая совместность $k$-го элемента описнвается уравнением 


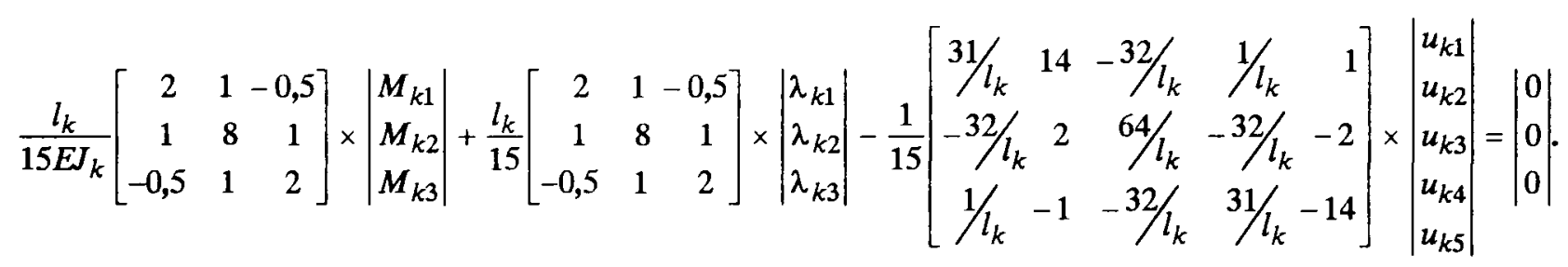

Матрищы $\left[D_{k}\right]$ и $\left[A_{k}\right]^{T}$ огределяются по формулам (10) и (11). Геометрические уравнения узлов таковн:

$$
-\lambda_{a}+u_{12}=0, \quad-\lambda_{b}-u_{15}+u_{22}=0, \quad \text { где } \quad \lambda_{a} \geq 0, \quad \lambda_{b} \geq 0 .
$$

Заданная функция изгибающих моментов удовлетворяет уравнению

$$
M_{k}(x)=\frac{1}{E J_{k}}\left\{[\infty]^{T} u_{k}(x)-\left[\Phi_{x}\right]^{T} \lambda_{k}(x)\right\} .
$$

Поэтому построенный конечный элемент является совместным и решение задачи можно определить согласно совместной математической модели (29) без предварительной аптроксимащии изгибающих моментов, которне после решения задачи (29) могут быть определенн по формуле (35). Все-тахи для решения задачи целесообразнее использовать математическую модель (32).

Получены следующие результаты расчета: а) множители $\lambda_{a}=0, \lambda_{b}=0.75 M_{0} / E J ; \lambda_{i}=0$, где $i=1,2, \ldots, 6 ; 6)$ векторы перемещений $\mathbf{u}_{r}=\{0,0.2411,-0.3214,0.4286,0.4821,-0.2143\}^{T} M_{0} / E J \quad$ и $\mathbf{u}=\{0,1,0.0833,3.0625,1.8333\}^{T} M_{0} / E J$; в) векторы изгибающих моментов $\mathbf{M}_{r}=\{-0.1071,0.0536$, $0.2143,0.2143,0.1071,0\}^{T} M_{0}$ и $\quad \mathrm{M}=\{-0.9166,0.4583,-1,-1,0.9166,0\}^{T} M_{0}$. На рис. $1.3-1.5$ похазаны эторы упругих, остаточных и полнш изгибаюших моментов. Численшые результаты совпадают с результатами аналитического решения балки.

\section{6. Заклотенас}

На основе экстремальных энергетических принципов и смешанных конечных элементов построены дискретные смешанные и статические постановки задач анализа полных и остаточных перемещений и напряжений упругопластических конструхций при разншх видах нагружения. Они представляют собой двойственную пару задач математического программирования. В них учитываются возможные разрывы перемещений и диссипашия энергии на поверхностях раздела конечных элементов. Представлены дискретные выражения условий текучести и геометрических уравнений. Похазано, что, во-первьх, метод Бубнова-Галерхина дает наитушую аппрохсимацио геометрических уравнений, пластических деформаций, а заодно и энергии деформаций, в том числе диссипативной энергии. Во-вторнх, применение точечных условий текучести в двойственных постановхах задач означает применение различных способов колшюкаций для аптроксимации упругих и пластических деформаций. Упругие деформащии и упругая энергия апшрохсимируются методом Бубнова-Галеркина, а пластические деформации - методом точечной коллокации. Чтобы избежать такое несоответствие и повысить точность результатов расчета для дискретизации функапональньх и дифференциальнкх зависимостей, следует применять метод коллохации Бубнова-Галеркина.

Для случая линейных условий текучести построены также кинематические постановхи задачи анализа остаточнох перемещений и напряжений. Неизвестньми в них являются лишь пластические множители. Получены зависимости остаточных перемещений и напряжений от пластических множителей. Смешанные и кинематические постановки задач соответствуют экстремальному 
принииу о минимуме полной энергия деформаций и позволяют определшть нжжною оценку параметров НДС. Вместе с равновесными математическими моделями, представленными в [3], они позволяют огределить двухстороннюю оценку НДС упругопластических конструкций и, таким образом, позволяют оценить точность численных результатов расчета. Построение и применение смешанных и совместных конечных элементов иллюстрируется примером анализа НДС балки.

Разработанная методика учета разрывов фупкций перемещений применительно к простому нагружению может быть использована и в других задачах упругопластическопо анализа, а также для более сложного повторно--переменного иш подвижного нагружения.

\section{Лгтерхтура}

1. А.А. Чирас. Математические модели анализа и огтимизации упругопластических систем. Вильнюс: Мокслас, 1982. 112 c.

2. А.А. Чирас. Теория оптимизации в предельном анализе твердого деформируемого тела. Вильнюс: Минтис, 1971. 123 с.

3. С. Каланта. Двойственные математические модели задач анализа упругопластических конструкций с разрывными полями перемещений// Statyba (Строительство), № 3(3), Вильнюс: Техника, 1995, с. 5-29.

4. А.В. Сокас. Определение напряженно-деформированного состояния идеально упругопластических сферических оболочек при несимметрчном простом нагрухении до достихения пластического разрушения. Автореферат дисс. на соискание ученой степени канд. техн. наук, Вильнюс, 1986. 16 с.

5. Ю.Ю. Аткочюнас. Расчет упрупопластических систем при повторных нагружениях. Вильнюс: Изд-во науки и энциклопедий, 1994. $150 \mathrm{c}$.

6. T. Belytschko, M. Velebit. Finite element method for elastic plastic plates //J. Eng. Mech. Div. Proc. Amer. Soc. Civ. Eng., 98, № 1, 1972, p. 227-242.

7. T. Belytschko, P.G. Hodge. Plane stress limit analysis by finite element // J. Eng. Mech. Div. Proc. Amer. Soc. Civ. Eng., 96, № 6, 1970, p. 931-944.

8. A. Biron, G. Charleux. Limit analysis of axisymmetric pressure vessel intrsections of arbitrary shape // Int. J. Mech. Sci., 1972, 14, № 1, 1972, p. 25-41.

9. S. Turgeman, J. Pastor. Limit analysis: a linear formulation of the kinematic approach for axisymmetric mechanics problems //J. Int. Numer. and Anal. Meth. Geomech., 6, № 1, 1982, p. 109-128.

10. С.А. Каланта. Двойственные задачи предельного равновесия с разрывами скоростей перемещений // Statyba (Строительство), № 2(2), Вильнюс: Техника, 1995, с. 20-25.

11. Р. Галлагер. Метод конечных элементов. Основы. М.: Мир, 1984. 428 с. (пер. с англ.)

12. S. Kalanta. Takumo sąlygos baigtiniu elementy modeliams // Proceedings of 4th Int. Conf. "Modern building materials, structures and techniques", vol. III. Vilnius: Technika, 1995, p. 260-265.

İteikta 19960328

\section{MIŠRIEJI IR DARNIEJI BAIGTINIAI ELEMENTAI TAMPRIŲ-PLASTINIU KONSTRUKCIJU ANALIZĖS UŽDAVINIUOSE}

\section{S. Kalante}

Santra u a

Nagrinèjami idealiai tamprių-plastinių konstrukcijų, veikiamų ivairių išorinių poveikių (apkrovos, išankstinio deformavimo, atramų sédimo), įtempimų-deformacijų būvio analizès uždaviniai. Juose nustatomi konstrukcijos poslinkiai ir įtempimai bei jų liekamosios dalys. Remiantis ekstreminiais energetiniais principais, dualumo teorija ir baigtiniu elementu metodu sudaryti dualūs bendri ir diskretiniai šių uždaviniu matematiniai modeliai. Ju naujumas - įvertinami galimi poslinkiu funkcijų trūkiai ir energijos disipacija plastiniuose paviršiuose tarp baigtinių elementu.

Panaudojant mišruji energetini funkcionalą ir mišriuosius baigtinius elementus, pirmiausia sudarytos pagrindinių priklausomybių (geometrinių lygčių, takumo sąlygų) diskretinès išraiškos ir mišrūs diskretiniai uždavinių matematiniai modeliai. Jie atitinka pilnutinès deformacijų energijos minimumo principui. Duali statinè uždavinių formuluotè sudaroma Lagranžo daugikliu metodu ir atitinka papildomosios energijos minimumo principui. Tiesinių takumo sąlygu atveju, panaudojant mišrius ir geometriškai darnius baigtinius elementus, sudarytos uždavinių kinematinès formuluotès, kuriose nežinomieji yra tik kinematiniai dydžiai. Sudaryti matematiniai modeliai leidžia nustatyti ịtempimų ir poslinkių apatines 
reikšmes. Parodyta, kad iš visu klasikinị kolokacijos metodų, kurie gali būti naudojami geometrinių lygčių ir takumo sąlygı diskretizacijai, tiksliausiai geometrinès lygtys, plastinès deformacijos ir energijos disipacija aproksimuojamos Bubnovo-Galiorkino metodu. Sijos skaičiavimo pavyzdžiu iliustruojamas mišraus ir darnaus baigtinio elemento sudarymas ir panaudojimas tamprių-plastiniu konstrukcijų analizei.

\section{MIXED AND COMPATIBLE FINITE ELEMENTS IN THE ANALYSIS PROBLEM OF ELASTOPLASTIC STRUCTURES}

\section{S. Kalanta}

\section{S u m m a ry}

A problem of ideal elastoplastic structures stress-strain field determination is considered The dual general and discrete mathematical models of analysis problem are made on the basis of the extremal energy principles and finite element method. In these models the possible discontinuity of displacements and the dissipation of energy in the place of those discontinuities, also the different external effects (load, initial strains and support settlements) are estimated.

At first, on the basis of the mixed functional and mixed finite elements the discrete expressions of fundamental relationships (geometric equations, yield conditions) and the discrete mathematical model of mixed formulation of the problem are made. This mathematical model corresponds to the minimum total energy principle for a kinematically admissible displacements. The dual static formulation of the problem is obtained by Lagrange's multipliers method; this corresponds to the minimum complementary energy principle. The kinematic formulation of the problem is obtained in the case of linear yield conditions. These mathematical models permit to determine the lower values of the stress and displacements of structures. It has shown that the approximation of geometric equations and yield conditions by BubnovGaliorkin's collocation method gives the more accurate results. 